\title{
Microvascular decompression for atypical hemifacial spasm: lessons learned from a retrospective study of 12 cases
}

\author{
*Jiang Liu, MD, PhD, ${ }^{1}$ Yue Yuan, MD, ${ }^{1}$ Ying Fang, MD, ${ }^{2}$ Li Zhang, MD, PhD, ${ }^{1}$ Xiao-Li Xu, MD, \\ Hong-Ju Liu, MD, ${ }^{1}$ Zhe Zhang, MD, ${ }^{1}$ and Yan-Bing Yu, MD, PhD' ${ }^{1}$ \\ Departments of ${ }^{1}$ Neurosurgery and ${ }^{2}$ International Medicine, China-Japan Friendship Hospital, Beijing, China
}

\begin{abstract}
OBJECTIVE Typical hemifacial spasm (HFS) commonly initiates from the orbicularis oculi muscle to the orbicularis oris muscle. Atypical HFS (AHFS) is different from typical HFS, in which the spasm of muscular orbicularis oris is the primary presenting symptom. The objective of this study was to analyze the sites of compression and the effectiveness of microvascular decompression (MVD) for AHFS.

METHODS The authors retrospectively analyzed the clinical data for 12 consecutive patients who underwent MVD for AHFS between July 2008 and July 2013.

RESULTS Postoperatively, complete remission of facial spasm was found in 10 of the 12 patients, which gradually disappeared after 2 months in 2 patients. No recurrence of spasm was observed during follow-up. Immediate postoperative facial paralysis accompanied by hearing loss occurred in 1 patient and temporary hearing loss with tinnitus in 2. All 3 patients with complications had gradual improvement during the follow-up period.
\end{abstract}

CONCLUSIONS The authors conclude that most cases of AHFS were caused by neurovascular compression on the posterior/rostral side of the facial nerve distal to the root entry zones. MVD is a safe treatment for AHFS, but the incidence of postoperative complications, such as facial paralysis and decrease in hearing, remains high.

http://thejns.org/doi/abs/10.3171/2015.3.JNS142501

KEY WORDS microvascular decompression; atypical hemifacial spasm; facial nerve; brainstem; auditory evoked potential; functional neurosurgery

$\mathrm{H}$ EMIFacial spasm (HFS) is a movement disorder characterized by intermittent and involuntary contractions of muscles innervated by the ipsilateral facial nerve. Typical HFS commonly initiates from the orbicularis oculi muscle to the orbicularis oris muscle, buccinator muscle, and/or platisma. Atypical HFS (AHFS) is rare, in which spasm usually extends from the orbicularis oris muscle upward to the buccinator muscle and orbicularis oculi muscle. ${ }^{16}$ Typical HFS is commonly due to facial nerve compression by blood vessels where the nerve courses into the root entry zone (REZ) in the brainstem. Jannetta et al. identified that the most common factor for HFS was vascular compression affecting the proximal portion of the facial nerve. ${ }^{5,7}$

Kuroki et al. reported that both arterial compression and local demyelination of a branch of the peripheral facial nerve caused abnormal muscle responses in the rat, which suggests that the REZ is not the sole site of com- pression responsible for HFS. ${ }^{11}$ Slight injury to the myelin sheath of a peripheral portion of the facial nerve could make the nerve susceptible to vascular compression. ${ }^{11} \mathrm{Ryu}$ et al. reported on 2 of 155 patients (1.3\%) with AHFS who had distal compression on the posterior/rostral side of the facial nerve. ${ }^{16}$ So far, the mechanism of AHFS with distal neurovascular compression is still unknown. We report on 12 patients with AHFS. In addition, we retrospectively analyzed the compression sites of the facial nerve, and we discuss the pathophysiology and efficiency of microvascular decompression (MVD) for the treatment of AHFS.

\section{Methods \\ Patient Population}

Between July 2008 and July 2013, 12 consecutive patients with AHFS undergoing MVD at China-Japan Friendship Hospital were included in this study. There

ABBREVIATIONS AICA = anterior inferior cerebellar artery; AHFS = atypical HFS; HFS = hemifacial spasm; LSR = lateral spread response; MVD = microvascular decompression; $\mathrm{PICA}=$ posterior inferior cerebellar artery; $\mathrm{REZ}=$ root entry zone; $\mathrm{VA}=$ vertebral artery.

SUBMITTED November 2, 2014. ACCEPTED March 27, 2015.

INCLUDE WHEN CITING Published online September 4, 2015; DOI: 10.3171/2015.3.JNS142501.

* Drs. J. Liu and Yuan contributed equally to this work. 
were 4 males and 8 females, with a mean age of 51 years (range 34-62 years). The duration of symptoms ranged from 2 to 23 years with a mean of 13.5 years. The right side was affected in 5 patients and the left in 7 patients. Five of the 12 patients suffered from hypertension and 3 had diabetes. In addition, 2 patients had undergone 1 or more previous botulinum toxin injections leading to mild facial muscle paralysis. No patient had undergone botulinum toxin injection within 6 months preoperatively. The diagnosis of AHFS was based on the atypical symptom and physical examination. As a regular procedure, all patients underwent preoperative MRI to exclude other diseases.

\section{Intraoperative Monitoring}

Each patient was monitored by electromyography during the operation. Continuous facial electromyography monitoring was performed throughout the operation. Depolarizing muscle relaxants were not used during the operation. The stimulating needle electrodes were inserted intradermally over the zygomatic branches of the facial nerve, and a $0.3-\mathrm{msec}$ pulse wave with an intensity of 5-30 $\mathrm{mA}$ was used. ${ }^{8}$ When the nerve in charge of the frontalis muscle was stimulated, a lateral spread response (LSR) appeared in other facial muscles, including the orbicularis oculi, the orbicularis oris, and the mentalis muscles. During decompression of the offending vessels, the LSR may decrease in amplitude or frequency. If the LSR remained unstimulated even when increasing the stimulation intensity to the maximal value, we considered the facial nerve to be completely decompressed. ${ }^{8}$

\section{Operative Procedures}

A standardized lateral suboccipital retrosigmoid approach was performed in all patients as described previously. ${ }^{13}$ After induction of general anesthesia, the patient was placed in the contralateral decubitus position with the head rotated approximately $10^{\circ}$ away from the affected side and the vertex dropped $15^{\circ}$ toward the floor. This makes the retromastoid area the highest point in the surgical field. ${ }^{13}$ A conventional suboccipital retrosigmoid incision was made with a $2 \times 2-\mathrm{cm}$ bone window. ${ }^{13}$ After the edge of the sigmoid sinus was identified, the dura mater was opened and suspended to allow intermittent release of cerebrospinal fluid, which decreased intracranial pressure and provided enough space for microinstruments to enter. Using the microscope, the arachnoid membrane was dissected initially from the caudal cranial nerves. Careful dissection of the arachnoid membrane was performed throughout the cerebellopontine angle cistern, and gentle retraction of the cerebellum was obtained by an aspirator. The entire intracranial segment of the facial nerve was thus exposed during surgery. Meanwhile, any involved arteries were removed from the nerve by Teflon pads. Finally, the dura mater was closed with sutures in a watertight pattern, without placement of drainage.

\section{Clinical Evaluation}

Evaluation was performed when the patient had recovered from general anesthesia. After discharge, the patients were followed up by telephone and/or visits to an outpatient clinic. The postoperative follow-up duration ranged from 1.5 to 6.5 years (mean 4.5 years). Eight patients were followed up by telephone, and 4 patients were followed up in person. The efficacy of the operation was assessed using a new scoring system through combining the cure rate of symptoms with the complication rate. ${ }^{10}$ This new system reported by Kondo et al. in 2012 allows much more objective analysis of the results, improves the ability to discuss the results with others, and enhances comparability among different centers and institutes (Table 1). ${ }^{10}$ An evaluation of the overall results of MVD should be performed more than 1 year after surgery.

TABLE 1. Evaluation of MVD results for HFS*

\begin{tabular}{cl}
\hline \multicolumn{1}{c}{ Grade } & \multicolumn{1}{c}{ Definition } \\
\hline Evaluation of postoperative grade of involuntary movement $(\mathrm{E})$ & \\
\hline E0 & Complete disappearance of spasm \\
\hline E1 & Occasional slight spasm \\
\hline E2 & Moderate spasm, apparently persisting \\
\hline Evaluation of postoperative complications $(C)$ & Not cured \\
\hline C0 & No or only slight possible cranial nerve dysfunction \\
\hline C1 & Slight cranial nerve or cerebellar dysfunction, not bothersome \\
\hline C2 & for daily life \\
\hline Total evaluation of surgical results $(T)$ & Both subjective \& objective cranial nerve or cerebellar dysfunc- \\
\hline T0 & tion, problematic for daily life \\
\hline T1 & Excellent \\
\hline T2 & Good \\
\hline T3 & Fair \\
\hline
\end{tabular}

* Based on the system devised by Kondo et al. 
TABLE 2. Offending vessel and compression site

\begin{tabular}{llc}
\hline Vessel & \multicolumn{1}{c}{ Compression Site } & $\begin{array}{c}\text { No. of } \\
\text { Patients (\%) }\end{array}$ \\
\hline AICA & $\begin{array}{c}\text { DC in } 5 \text { patients; MC in 1 patients; MC+PC } \\
\text { in 1 patient }\end{array}$ & $7(58.3)$ \\
PICA & $\begin{array}{c}\text { PC in 1 patient; MC in 2 patients; DC in 1 } \\
\text { patient }\end{array}$ & $4(33.3)$ \\
VA+PICA & PC in 1 patient & $1(8.3)$ \\
\hline
\end{tabular}

$\mathrm{DC}=$ distal compression; $\mathrm{MC}=$ midportion compression; $\mathrm{PC}=$ proximal compression.

\section{Results}

\section{Operative Findings}

The offending vessels (Tables 2 and 3 ) were as follows: anterior inferior cerebellar artery (AICA) alone in 7 patients $(58.3 \%)$, posterior inferior cerebellar artery (PICA) alone in 4 patients $(33.3 \%)$, and the vertebral artery (VA) plus the PICA in 1 patient $(8.3 \%)$. The site of compression (Tables 2 and 3 ) was the REZ in 2 patients, the distal portion of the seventh cranial nerve around the internal auditory canal in 6 patients (Figs. 1 and 2), the middle portion of the nerve located between the REZ and the internal auditory canal in 3 patients (Fig. 3), and the REZ and middle portion of the nerve coexisting in 1 patient (Fig. 4). ${ }^{17}$ The characteristics of the case (Case 3 ) in which the REZ was compressed by the VA and PICA (Fig. 5) are consistent with those seen in typical hemifacial spasm as described by Mathur et al. ${ }^{12}$

\section{Postoperative Outcome}

Ten of the 12 patients $(83.3 \%)$ had relief of spasm immediately after surgery. The score involving the efficacy of surgery (E) was E0 (Table 1). The remaining 2 patients (16.7\%) had slight spasm, which diminished within 2 months postoperatively. The score involving the efficacy of surgery was E1. During the follow-up period, all 12 patients reported complete relief of facial spasm without other interventional therapies.

Two patients experienced pure-tone hearing loss with a high-pitched tinnitus immediately after surgery, which was reduced to $60 \mathrm{~dB}$. The complication score (C) involving these 2 patients was $\mathrm{Cl}$. Administration of prostaglandin, nimodipine, and neurotrophic agents (cobamamide and methycobal) improved hearing in these 2 patients. One patient suffered facial paralysis with tinnitus immediately postoperatively, which was also rectified through prostaglandin, nimodipine, and neurotrophic drugs (cobamamide and methycobal) for 1 month. The complication score for this patient was also $\mathrm{C} 1$. All 3 patients received hyperbaric oxygen therapy regularly after discharge, which gradually alleviated their symptoms. Although the tinnitus persists, it does not influence the patients' daily work and life. Preoperative mild facial muscle atrophy caused by botulinum toxin injection in 2 patients had no change after surgery. No postoperative intracranial infection or cerebrospinal fluid rhinorrhea was observed in our 12 patients.

The total evaluation of the results $(\mathrm{T})$ is judged by combining the $\mathrm{E}$ and $\mathrm{C}$ scores as explained in Table 3 . In our cases, 8 patients $(66.7 \%)$ achieved excellent results (T0), 3 patients $(25 \%)$ had good results (T1), and 1 patient $(8.3 \%)$ had fair results $(\mathrm{T} 2)$.

\section{Discussion}

In general, HFS is suggested to be the result of vascular compression. ${ }^{3}$ Campbell and Keedy ${ }^{2}$ and Gardner ${ }^{4}$ had reported that vascular compression of the facial nerve was the potential mechanism of HFS. However, they never mentioned the site of compression to the nerve. In 1977,

TABLE 3. Summary of the 12 cases and previously reported cases with AHFS

\begin{tabular}{|c|c|c|c|c|c|c|}
\hline $\begin{array}{l}\text { Case } \\
\text { No. }\end{array}$ & $\begin{array}{l}\text { Age (yrs), } \\
\text { Sex }\end{array}$ & Side & $\begin{array}{l}\text { Symptom Duration } \\
\text { (yrs) }\end{array}$ & Offending Vessel & Compression Site & Treatment/Outcome \\
\hline 1 & $34, F$ & Left & 15 & AICA & DC & Excellent \\
\hline 2 & $47, \mathrm{~F}$ & Left & 5 & AICA & $D C$ & Good $(\mathrm{E} 1+\mathrm{C} 0)$ \\
\hline 3 & $59, \mathrm{M}$ & Left & 23 & VA+PICA & REZ & Excellent \\
\hline 4 & $62, \mathrm{~F}$ & Right & 2 & PICA & DC & Good (E0+C1) \\
\hline 5 & $48, M$ & Left & 20 & PICA & REZ & Excellent \\
\hline 6 & $55, \mathrm{~F}$ & Right & 15 & AICA & REZ+MC & Good (E0+C1) \\
\hline 7 & $42, \mathrm{M}$ & Right & 6 & AICA & $\mathrm{MC}$ & Excellent \\
\hline 8 & $47, \mathrm{~F}$ & Left & 12 & AICA & DC & Excellent \\
\hline 9 & $52, F$ & Left & 19 & PICA & MC & Excellent \\
\hline 10 & $57, \mathrm{~F}$ & Right & 21 & AICA & DC & Excellent \\
\hline 11 & $49, M$ & Right & 16 & PICA & MC & Excellent \\
\hline 12 & $60, F$ & Left & 8 & AICA & DC & Fair (E1+C1) \\
\hline $13^{*}$ & $59, \mathrm{~F}$ & Left & 7 & PICA+AICA & REZ+MC & Good (E0+C1): 3 operations (MVD) \\
\hline $14^{*}$ & $36, \mathrm{~F}$ & Left & 6 & AICA & DC & Excellent \\
\hline $15 \dagger$ & $32, \mathrm{M}$ & Left & 1.5 & VA $\ddagger$ & REZ & Conservative therapy \\
\hline
\end{tabular}



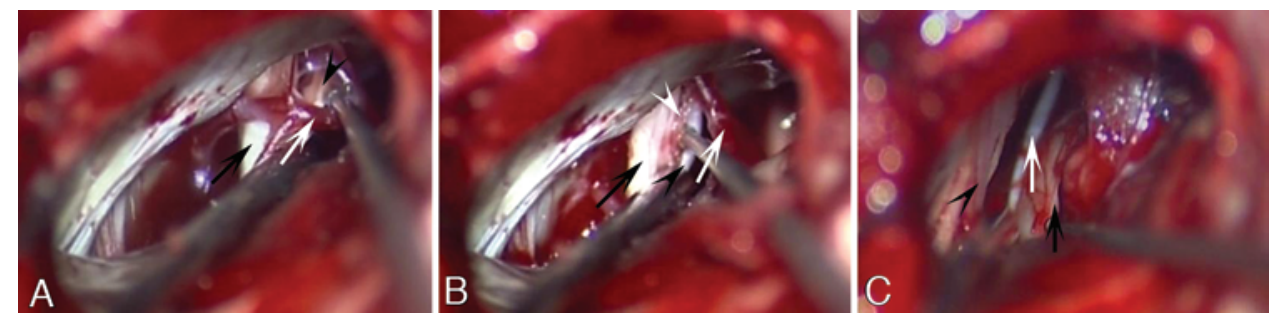

FIG. 1. Intraoperative photographs obtained in a patient with left AHFS, revealing compression of the facial nerve at the distal area only. A: The offending vessel was the AICA (white arrow), which coursed between the facial nerve (arrowheads) and cochlear nerve (black arrow in A and B). B: Teflon pads (white arrowhead) were inserted between the AICA and facial nerve (black arrowheads). C: Intraoperative photograph of the relationship of the glosspharyngeal nerve (arrowhead), abducent nerve (white arrows), and REZ (black arrow). There was no vessel at the REZ (black arrow). Figure is available in color online only.

Jannetta et al. ${ }^{6}$ first demonstrated that vascular compression to the REZ of the facial nerve was the cause of HFS. For the treatment of HFS, most neurosurgeons paid more attention to the REZ of the facial nerve and ignored vascular compression to this nerve at other sites. HFS was divided into typical HFS and AHFS according to onset sequence of associated muscles. Barker et al. ${ }^{1}$ reported that $8 \%$ of 648 patients with HFS presented with AHFS, but the authors did not offer an explanation of the pathophysiology of AHFS. Until now, only 3 cases of AHFS had been reported, 2 in Japan ${ }^{14}$ and 1 in England. ${ }^{16}$ We successfully diagnosed 12 patients as having AHFS. We concluded that the meatal loop of the AICA was the main offending vessel in most cases, which is consistent with other reports. With respect to the compression site, the outcomes of our study were variable. The entire intracranial segment of the facial nerve can be classified into 3 portions, including proximal (REZ), middle (between the REZ and the internal auditory canal), and distal (around the internal auditory canal). ${ }^{17}$ Among our 12 patients, 2 patients suffered from proximal compression, 6 distal, 3 middle, and 1 both REZ and middle.

There are few studies reporting the compression sites and clinical outcomes of MVD for AHFS. Ryu et al. ${ }^{16}$ reported 2 of 155 cases (1.3\%) with AHFS, one of which was caused by vascular compression of a distal portion and REZ of the facial nerve, while the other was caused by the distal portion of the nerve alone. The intraoperative findings suggested that the nerve fibers at the anterior/cau-

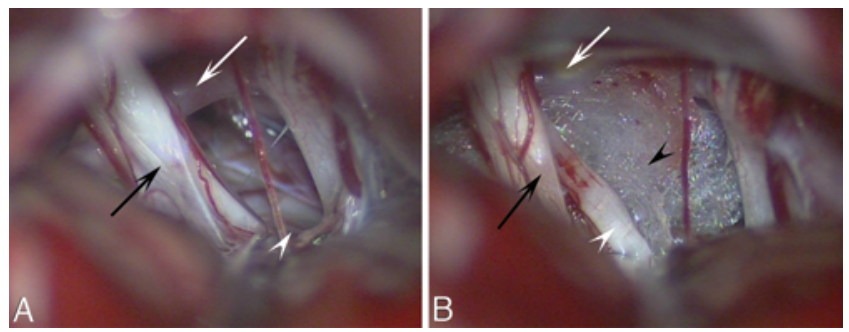

FIG. 2. Intraoperative photographs obtained in a patient with right AHFS, revealing compression of the facial nerve at the distal area only. A: There was no vessel at the REZ (arrowhead). The PICA was the offending vessel (white arrow), which compressed the eighth cranial nerve (black arrow) from the ventral side. B: Intraoperative photograph of the relationship of the facial nerve (white arrowhead), auditory nerve (black arrow), and PICA (white arrow). Teflon pads (black arrowhead) were inserted between the PICA and facial nerve (white arrowhead). Figure is available in color online only. dal side of the facial nerve in the cerebellopontine cistern innervate the upper part of the facial muscles (the orbicularis oculi and frontalis muscles), while the fibers at the posterior/rostral side of the nerve innervate the lower part of the facial muscles (the orbicularis oris and buccinator muscles). ${ }^{16}$ They concluded that the facial nerve seems to be rotated along its course between the cerebellopontine cistern and the exit of the facial canal, which explained the possible mechanisms of AHFS based on topography. The main compression site in most of our cases was distal to the REZ of the facial nerve. These findings are similar to those in the report of Ryu et al. Although most compression sites were different from those of typical HFS, all 12 patients did not suffer from HFS after the offending vessels were detached from the facial nerve.

The mechanism of AHFS attributed to compression of distal portions of the seventh cranial nerve is still unknown. The present findings of our study demonstrate that distal compression of the seventh cranial nerve by blood vessels plays an important role. ${ }^{17}$ Partial demyelination and axonal degeneration of the seventh cranial nerve in typical HFS with neurovascular compression have been also reported. ${ }^{15}$ The same mechanism might be appropriate for interpreting the cause of AHFS with compression of a distal portion of the seventh cranial nerve. Schwann cells are more resistant to vascular compression in demyelination. Thus, we concluded that more force might be produced in inducing HFS at the distal area of the facial nerve. ${ }^{17}$ As such, the incidence rate of AHFS is less than that of typical HFS.
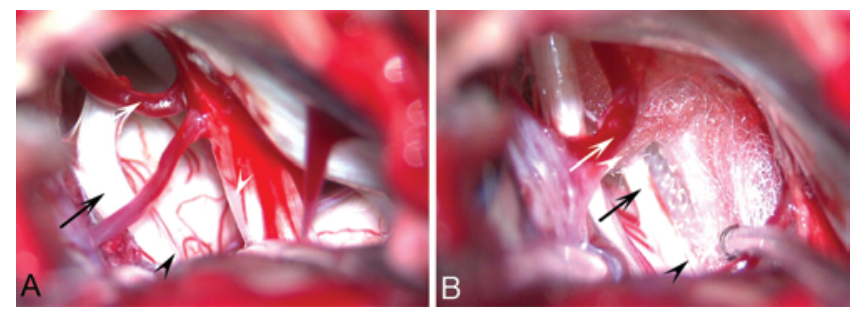

FIG. 3. Intraoperative photographs obtained in a patient with right AHFS, revealing compression of the facial nerve at the midportion area only. A: There was no vessel at the REZ (black arrowhead) near the glosspharyngeal nerve (white arrowhead). The AICA was the offending vessel (white arrow), which penetrated between the facial nerve (black arrow) and auditory nerve. B: There was no vessel at the REZ (black arrowhead). Teflon pads (white arrowhead) were inserted between the AICA (white arrow) and facial nerve (black arrow). Figure is available in color online only. 

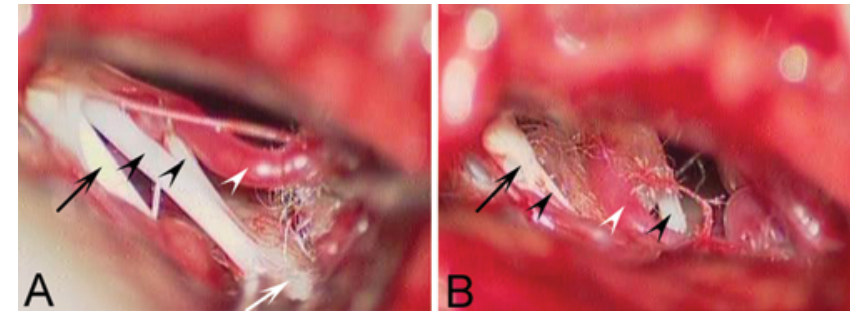

FIG. 4. Intraoperative photographs obtained in a patient with right AHFS, revealing that the facial nerve was compressed at both the midportion area and the REZ. A: The AICA was the offending vessel (white arrowhead), which penetrated through the facial nerve (black arrowheads), and not between facial nerve and auditory nerve (black arrow). Teflon pads were inserted between the AICA (white arrowhead) and brainstem at the REZ (white arrow). B: Teflon pads were inserted between the AICA (white arrowhead) and facial nerve (black arrowheads) at the midportion area of facial nerve, and not between the AICA and auditory nerve (black arrow). Figure is available in color online only.

When facing patients with HFS, every neurosurgeon should carefully take the patient's history to identify typical or atypical HFS. Once a patient has been diagnosed as having AHFS, the distal portion of the facial nerve and the REZ should be simultaneously examined. ${ }^{16}$ To obtain excellent or good results, the seventh cranial nerve should be sufficiently decompressed despite the high risk of postoperative facial weakness or hearing disturbance. The offending vessels in 7 patients of our study were the meatal branch of the AICA which penetrated between the facial and cochlear nerves. To decompress the facial nerve sufficiently, Teflon pads were placed between the offending vessel and the facial nerve. Considering the vulnerability of the facial nerve, this procedure may cause partial facial paralysis.

In addition, the incidence of tinnitus and hearing loss occurring in our patients was relatively high. The AICA is the major feeding artery that sends branches to support the cochlear nerve. ${ }^{16}$ Due to narrowed operative space, the offending vessel may cause vasospasm, leading to insufficient blood supply for the facial nerve. On the other hand, Teflon pads inserted between the offending vessel and facial nerve may disturb the microcirculation of the internal auditory meatus. Therefore, we placed Teflon pads between the offending vessel and the pons, or we attached the offending vessel to adjacent dura mater with glue in patients with the offending vessel located at the REZ of the facial nerve. ${ }^{16}$ In our patients with distal compression, we simply placed Teflon pads between the offending vessel and facial nerve. LSR was used to evaluate the efficiency of MVD in our study. ${ }^{13}$ However, in one of our patients, no complete disappearance in the amplitude and frequency of LSR was observed even when repeatedly adjusting the position of the Teflon pads. In addition, real-time electrophysiological monitoring was crucial in determining the proper placement of the Teflon pads. ${ }^{9}$ During follow-up, all 12 patients were free of facial spasm, supporting the correct position of the Teflon pads and the significance of continuous monitoring during surgery.

\section{Conclusions}

In this study, the importance of history taking was raised for diagnosis of atypical HFS. Complete decompression of the facial nerve is necessary in patients with AHFS. In addition, real-time electrophysiological monitoring plays a considerable role in the identification of offending vessels, as well as the navigation of the operation in verifying the decompression of facial nerve. Finally, MVD remains the most effective therapy for AHFS.

\section{Acknowledgment}

This work was supported by grants from the China-Japan Friendship Hospital Youth Science and Technology Excellence Project (2015-QNYC-B-04 and 2014-QNYC-A-05).

\section{References}

1. Barker FG II, Jannetta PJ, Bissonette DJ, Shields PT, Larkins MV, Jho HD: Microvascular decompression for hemifacial spasm. J Neurosurg 82:201-210, 1995

2. Campbell E, Keedy C: Hemifacial spasm; a note on the etiology in two cases. J Neurosurg 4:342-347, 1947

3. Chung SS, Chang JH, Choi JY, Chang JW, Park YG: Microvascular decompression for hemifacial spasm: a long-term follow-up of 1,169 consecutive cases. Stereotact Funct Neurosurg 77:190-193, 2001

4. Gardner WJ: Concerning the mechanism of trigeminal neuralgia and hemifacial spasm. J Neurosurg 19:947-958, 1962

5. Jannetta PJ: Arterial compression of the trigeminal nerve at the pons in patients with trigeminal neuralgia. 1967. J Neurosurg 107:216-219, 2007

6. Jannetta PJ: Observations on the etiology of trigeminal neuralgia, hemifacial spasm, acoustic nerve dysfunction and glossopharyngeal neuralgia. Definitive microsurgical treatment and results in 117 patients. Neurochirurgia (Stuttg) 20:145-154, 1977
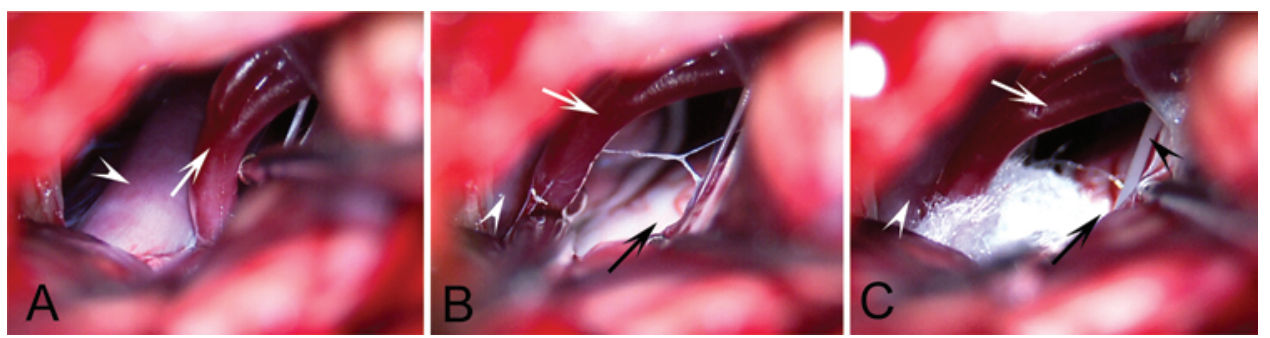

FIG. 5. Intraoperative photographs obtained in a patient with left atypical hemifacial spasm, revealing compression of the facial nerve at the REZ. A: The VA (white arrowhead) and the PICA (white arrow) were the offending vessels, which typically compressed at the REZ. B: The VA (white arrowhead) and PICA (white arrow) were lifted from the REZ (black arrow). C: Teflon pads were inserted the between VA (white arrowhead) and the PICA (white arrow) and facial nerve (black arrowheads) at the REZ (black arrow) of the facial nerve. Figure is available in color online only. 
7. Jannetta PJ, Abbasy M, Maroon JC, Ramos FM, Albin MS: Etiology and definitive microsurgical treatment of hemifacial spasm. Operative techniques and results in 47 patients. J Neurosurg 47:321-328, 1977

8. Kang MC, Choi YS, Choi HK, Lee SH, Ghang CG, Kim $\mathrm{CH}$ : Efficacy of the disappearance of lateral spread response before and after microvascular decompression for predicting the long-term results of hemifacial spasm over two years. J Korean Neurosurg Soc 52:372-376, 2012

9. Kim HJ, Park YS, Ryu JS, Huh R, Han I, Shin DA, et al: Intraoperative facial electromyography and brainstem auditory evoked potential findings in microvascular decompression for hemifacial spasm: correlation with postoperative delayed facial palsy. Stereotact Funct Neurosurg 90:260-265, 2012

10. Kondo A, Date I, Endo S, Fujii K, Fujii Y, Fujimaki T, et al: A proposal for standardized analysis of the results of microvascular decompression for trigeminal neuralgia and hemifacial spasm. Acta Neurochir (Wien) 154:773-778, 2012

11. Kuroki A, Møller AR: Facial nerve demyelination and vascular compression are both needed to induce facial hyperactivity: a study in rats. Acta Neurochir (Wien) 126:149-157, 1994

12. Mathur T, Srivastava T, Sardana V, Jain R: Atypical hemifacial spasm due to vertebral artery dolichoectasia: rare cause of a rarer clinical entity. BMJ Case Rep 2013:bcr2013010199, 2013

13. McLaughlin MR, Jannetta PJ, Clyde BL, Subach BR, Comey $\mathrm{CH}$, Resnick DK: Microvascular decompression of cranial nerves: lessons learned after 4400 operations. J Neurosurg 90:1-8, 1999

14. Nikaido H, Kobayashi E, Nagata K, Kayahara T: [A case of atypical hemifacial spasm without involvement of orbicularis oculi muscles: possibility of functional topography in facial nerve.] No Shinkei Geka 23:73-77, 1995 (Jpn)

15. Ruby JR, Jannetta PJ: Hemifacial spasm: ultrastructural changes in the facial nerve induced by neurovascular compression. Surg Neurol 4:369-370, 1975

16. Ryu H, Yamamoto S, Miyamoto T: Atypical hemifacial spasm. Acta Neurochir (Wien) 140:1173-1176, 1998

17. Ryu H, Yamamoto S, Sugiyama K, Uemura K, Miyamoto $\mathrm{T}$ : Hemifacial spasm caused by vascular compression of the distal portion of the facial nerve. Report of seven cases. $\mathbf{J}$ Neurosurg 88:605-609, 1998

\section{Disclosure}

The authors report no conflict of interest concerning the materials or methods used in this study or the findings specified in this paper.

\section{Author Contributions}

Conception and design: Yu, J Liu, Z Zhang. Acquisition of data: J Liu, Yuan, Fang, Z Zhang. Analysis and interpretation of data: J Liu, Yuan, Fang, L Zhang, Xu, H Liu. Drafting the article: J Liu. Critically revising the article: Yu. Reviewed submitted version of manuscript: Yuan, L Zhang, H Liu. Statistical analysis: L Zhang, Xu, H Liu, Z Zhang. Administrative/technical/material support: Yuan, L Zhang, Xu. Study supervision: Yu, Yuan.

\section{Correspondence}

Yanbing Yu, Department of Neurosurgery, China-Japan Friendship Hospital, No. 2, Yinghua East St., ChaoYang District, Beijing 100029, China. email: yuyanbing123@126.com. 